\title{
Outer-Membrane-Vesicle-Associated O Antigen, a Crucial Component for Protecting Against Bordetella parapertussis Infection
}

\author{
Daniela Bottero ${ }^{1}$, María Eugenia Zurita ${ }^{1}$, María Emilia Gaillard ${ }^{1}$, \\ Francisco Carriquiriborde ${ }^{1}$, Pablo Martin Aispuro ${ }^{1}$, Maia Elizagaray ${ }^{2}$, Erika Bartel ${ }^{1}$, \\ Celina Castuma ${ }^{1}$ and Daniela Hozbor ${ }^{1 *}$
}

${ }^{1}$ Laboratorio VacSal, Facultad de Ciencias Exactas, Instituto de Biotecnología y Biología Molecular, Universidad Nacional de La Plata, La Plata, Argentina, ${ }^{2}$ Facultad de Ciencias Exactas, Instituto de Estudios Inmunológicos y Fisiopatológicos, Universidad Nacional de La Plata, La Plata, Argentina

OPEN ACCESS

Edited by:

Fabio Bagnoli,

GlaxoSmithKline, Italy

Reviewed by:

Giuseppe Lofano,

GSK Vaccines, United States

Ulrike Resch

Medizinische Universität Wien, Austria

Dipankar Ghosh,

Jawaharlal Nehru University, India

*Correspondence:

Daniela Hozbor

hozbor.daniela@gmail.com;

hozbor@biol.unlp.edu.ar

Specialty section:

This article was submitted to Vaccines and Molecular Therapeutics, a section of the journal

Frontiers in Immunology

Received: 03 May 2018 Accepted: 10 October 2018

Published: 29 October 2018

Citation:

Bottero D, Zurita ME, Gaillard ME, Carriquiriborde F, Martin Aispuro P,

Elizagaray $M$, Bartel E, Castuma $C$ and Hozbor D (2018)

Outer-Membrane-Vesicle-Associated O Antigen, a Crucial Component for

Protecting Against Bordetella

parapertussis Infection.

Front. Immunol. 9:2501.

doi: 10.3389/fimmu.2018.02501
Bordetella parapertussis is a respiratory-disease pathogen producing symptomatology similar to that of pertussis but of underestimated incidence and with no specific vaccine existing. We recently designed a vaccine candidate from $B$. parapertussis outer-membrane vesicles (OMVs) that proved to be safe and protective in a murine-infection model. Based on protection recently reported for the B. parapertussis $O$ antigen in aqueous solution, we assessed here whether the $B$. parapertussis O-antigen-containing lipopolysaccharide (BppLPS-O ${ }^{+}$) embedded in the membranes, as present in $B$. parapertussis-derived OMVs (OMVs(Bpp-LPS-O $\left.{ }^{+}\right)$), was the component responsible for that previously observed protection by OMVs. By performing a comparative study with OMVs from a human strain with undetectable $\mathrm{O}$ antigen (OMVs(Bpp-LPS-O- $)$ ), we demonstrated that the OMVs(Bpp-LPS-O ${ }^{+}$), but not the OMVs(Bpp-LPS-O- ${ }^{-}$, protected mice against sublethal B. parapertussis infections. Indeed, the $B$. parapertussis loads were significantly reduced in the lungs of OMVs(Bpp-LPS-O $\left.{ }^{+}\right)$-vaccinated animals, with the CFUs recovered being decreased by 4 log units below those detected in the non-immunized animals or in the animals treated with the OMVs(Bpp-LPS-O- $),(p<0.001)$. We detected that the OMVs(Bpp-LPS-O $\left.{ }^{+}\right)$induced IgG antibodies against $B$. parapertussis whole-cell lysates, which immunocomponents recognized, among others, the $O$ antigen and accordingly conferred protection against B. parapertussis infection, as observed in in-vivo-passive-transfer experiments. Of interest was that the OMVs(Bpp-LPS-O ${ }^{+}$) -generated sera had opsonophagocytic and bactericidal capabilities that were not detected with the OMVs(Bpp-LPS-O- $)^{-}$-induced sera, suggesting that those activities were involved in the clearance of $B$. parapertussis. Though stimulation of cultured spleen cells from immunized mice with formulations containing the $O$ antigen resulted in gamma interferon (IFN- $\gamma$ ) and interleukin-17 production, spleen cells from OMVs(Bpp-LPS-O $\left.{ }^{+}\right)$-immunized mice did not significantly contribute to the observed protection against $B$. parapertussis infection. 
The protective capability of the $B$. parapertussis $O$ antigen was also detected in formulations containing both the OMVs derived from $B$. pertussis and purified BppLPS$\mathrm{O}^{+}$. This combined formulation protected mice against $B$. pertussis along with $B$. parapertussis.

Keywords: Bordetella parapertussis, outer-membrane vesicles, lipopolysacharides, O-antigen, protection

\section{INTRODUCTION}

Bordetella parapertussis is a species quite close to Bordetella pertussis that can infect humans causing similar symptoms to those of the respiratory disease referred to as pertussis. The detection of this pathogen in pertussis patients is relatively frequent in different countries of Europe $(1,2)$ and also in the USA (3-5). In the latter country, the highest number of B. parapertussis infections was recorded in Wisconsin (at 443 cases) between October 2011 and December 2012 (5). As had occurred previously, during such outbreaks $11.2 \%$ of the diagnostic specimens positive for Bordetella were also positive for $B$. parapertussis, probably indicating the endemnism of $B$. parapertussis. In fact, in the USA B. parapertussis was estimated to have caused $16 \%$ of the cases diagnosed as pertussis (6). In several countries of Latin America, infections caused by $B$. parapertussis have been detected, but unfortunately no official reports about the incidence rates are available. We wish to note here that in general the global incidence of $B$. parapertussis is probably underestimated, not only in LatinAmerican countries but also in most others because the official notification of the infections caused by this pathogen are not mandatory. Furthermore, many laboratories do not have the technologic wherewithal to discriminate between $B$. pertussis and $B$. parapertussis infections. In addition, $B$. parapertussis must clearly be recognized as the cause of a pertussis-like disease for which no specific effective preventive strategies have as yet been developed. Moreover, the currently used vaccines for pertussis are not adequate for reducing $B$. parapertussis infections (7). Several of the protective immunogens included in the pertussis vaccines, though homologous to $B$. parapertussis proteins, are antigenically distinct $(7,8)$. This may be one of the reasons that could explain the observed incomplete cross-protection of pertussis vaccines against $B$. parapertussis. Furthermore, these two species show differences in the expression of components that are essential for the protection induced by pertussis vaccines. As an example, the pertussis toxin is only expressed by $B$. pertussis and since this toxin is not present in B. parapertussis, the protective immune response triggered by the toxin has no target in B. parapertussis.

Only two studies have indicated that pertussis acellular vaccines could have any effect whatsoever on the control of $B$. parapertussis; and as described by the authors, those studies had several limitations-mainly as a consequence of the underreporting of $B$. parapertussis, which underestimation must necesarily affects the assessment of vaccine effectiveness $(2,9)$. Even worse, Long et al., using a rodent model of bordetellosis, demonstrated that pertussis acellular vaccination enhances the performance of B. parapertussis (10).
The ongoing research activity on $B$. parapertussis points to the demand for a specific vaccine against this pathogen. The proteins of $B$. parapertussis - such as the filamentous hemagglutinin and the iron-transport protein AfuA, among others-had been proven to confer protection against $B$. parapertussis infection in a mouse model $(11,12)$. In previous work, we started to investigate the potential of outer-membrane vesicles (OMVs) derived from $B$. parapertussis as an alternative approach to a vaccine candidate against $B$. parapertussis (13). Using the mouse model of intranasal infection, we observed that the formulations based on these OMVs efficiently protected mice against $B$. parapertussis infection, whereas current commercial acellular pertussis vaccines exhibited little protection against that particular pathogen (13). OMVs are naturally released by various Gram-negative bacteria and contain predominantly outer-membrane components, including the lipopolysaccharide (LPS), along with periplasmic compounds (14). That the isolated LPS of $B$. parapertussis-and in particular the LPS containing the $\mathrm{O}$ antigen (BppLPS- ${ }^{+}$)- has been found to protect against $B$. parapertussis infection is noteworthy (15). That protection was revealed by the assays carried out in a mouse model in which immunizations were performed with commercial acellular vaccines supplemented with an aqueous solution containing $10 \mu \mathrm{g}$ of purified BppLPS-O ${ }^{+}(15)$. That protective capability was, in fact, associated specifically with the presence of the $\mathrm{O}$ antigen (15). At this point, we must stress that though all B. parapertussis has the unusual lipid A structure characteristic to Bordetella [absence of symmetry at the C-3 and C-3' positions, phospate groups modified with glucosamine and hipo-acylation, $(16,17)]$, not all lineages of $B$. parapertussis contain an LPS whose structure includes the $\mathrm{O}$ antigen. The lineage that infects only humans contains an LPS with the $\mathrm{O}$ antigen, whereas the LPS of the strains that have been recovered from sheep lack that antigen (18). We were also interested to note that the isolates containing LPS without the $\mathrm{O}$ antigen are highly sensitive to murine complementmediated killing in vitro, and because of that vulnerability those B. parapertussis isolates failed to colonize naïve mice (18).

Within this context, in the present work, we assessed whether the $B$. parapertussis LPS with the $\mathrm{O}$ antigen embedded in the membranes-as occurs in the example of the OMVs derived from B. parapertussis OMVs(Bpp-LPS- $\left.{ }^{+}\right)$-would prove to be the crucial component for the previously reported protection of the OMVs (13). To assess the role of LPS-O ${ }^{+}$, the immunity and protection conferred by $B$. parapertussis-OMV vaccination were compared to the properties generated by the OMVs derived from an ATCC strain of B. parapertussis in which 
the $\mathrm{O}$ antigen was not detected (OMVs $\left.\left(\mathrm{Bpp}-\mathrm{LPS}_{-} \mathrm{O}^{-}\right)\right)$. Mice immunized with OMVs derived from a clinical isolate of $B$. parapertussis whose LPS contained the $\mathrm{O}$ antigen were protected against $B$. parapertussis, but not those treated with OMVs derived from $B$. parapertussis lacking that antigen. By performing in-vitro and in-vivo experiments, we detected that the humoral response containing $\mathrm{O}$-antigen-specific antibodies contributed to the protection induced by the OMVs(Bpp-LPS$\mathrm{O}^{+}$) -vaccines. In contrast, sera collected from OMVs(BppLPS- ${ }^{-}$) immunized mice produced no such reduction of B. parapertussis colonization upon passive transfer. Purified BppLPS-O $^{+}$, but not the BppLPS- ${ }^{-}$, induced protection in mice against $B$. parapertussis challenge. Moreover, the inclusion of $\mathrm{BppLPS}_{-}{ }^{+}$, but not $\mathrm{BppLPS}^{-} \mathrm{O}^{-}$, rendered the vaccine based on OMVs derived from B. pertussis (19-24) efficacious against both $B$. parapertussis and $B$. pertussis challenge. All together, these data indicated that $\mathrm{LPS}^{-} \mathrm{O}^{+}$is a crucial protective antigenic component of the OMVs derived from B. parapertussis.

\section{MATERIALS AND METHODS}

\section{Bacterial Strains and Growth Conditions}

Bordetella parapertussis AR729, an Argentine clinical isolate previously used to obtain the OMVs, along with the ATCC strain B. parapertussis 15237-the isolate from Eldering and Kendrick lacking the LPS $\mathrm{O}$ antigen-were used throughout this study (25). In contrast to other human B. parapertussis isolates, the ATCC strain has undetectable $\mathrm{O}$ antigen (18). These strains were grown either in Bordet-Gengou agar (BGA, Difco) supplemented with $1 \%(\mathrm{v} / \mathrm{v})$ glycerol, $10 \mathrm{~g} / \mathrm{L}$ Bacto peptone (Difco), and $10 \%(\mathrm{v} / \mathrm{v})$ defibrinated sheep blood and incubated at $36^{\circ} \mathrm{C}$, or alternatively in Stainer-Scholte liquid medium (26), as indicated previously (13).

\section{Isolation of OMVs}

To obtain OMVs from the bacterial cells, we used the method previously described by us $(19,20,27)$. The procedure stated in brief: Culture samples from the decelerating growth phase of the bacteria were centrifuged at $10,000 \mathrm{x}$ g for $20 \mathrm{~min}$ at $4^{\circ} \mathrm{C}$ and the pellet obtained resuspended in $20 \mathrm{mM}$ Tris- $\mathrm{HCl}, 2 \mathrm{mM}$ EDTA, pH 8.5 (TE Buffer). Of the resulting pellet, $\sim 1 \mathrm{~g}$ (wet weight) was resuspended in $5 \mathrm{~mL}$ of the TE Buffer. OMV release was promoted by sonication in ice-water; the cells were then removed by centrifugation at 10,000 $\mathrm{x}$ g and the OMV-containing supernatant concentrated by ultracentrifugation at 40,000 $\mathrm{x}$ g for $3 \mathrm{~h}$. The OMVs thus obtained were stored at $4^{\circ} \mathrm{C}$. Thereafter the OMVs were examined by electron microscopy after negative staining (20).

\section{Protein Assay}

The protein content was estimated by the Bradford method with BSA as a standard (28).

\section{LPS Sodium Dodecylsulfate-Polyacrylamide Gel Electrophoresis (SDS-PAGE) and Immunoblotting}

The LPS from the OMVs of $B$. parapertussis was solubilized in Laemmli sample buffer (29) and heated at $100^{\circ} \mathrm{C}$ for $10 \mathrm{~min}$. Twenty-five mg of proteinase $\mathrm{K}$ in $10 \mu \mathrm{L}$ of Laemmli sample buffer were added per $50 \mu \mathrm{L}$ of OMV suspension. The mixtures were incubated in a water bath at $60^{\circ} \mathrm{C}$ for $1 \mathrm{~h}$ with occasional vortexing. The proteinase-K-treated samples were applied to the gels and the electrophoresis performed at room temperature and constant voltage. The LPS visualized by the BioRad silver-staining technique was transferred to polyvinylidenedifluoride membranes (Immobilon P; Millipore) and probed with OMV-immune sera $(1: 2,000)$ followed by incubation with anti(mouse IgG) conjugated with alkaline phosphatase at a 1:1,000 dilution. Nitroblue tetrazolium and 5-bromo-4-chloro3-indolyl-phosphate were used as the phosphatase substrates according to the manufacturer's protocol (Biodynamics SRL Buenos Aires Argentina).

\section{Isolation of $B$. parapertussis LPS}

The LPS from B. parapertussis was isolated by the hot phenolwater method, along with previously described modifications (30). The samples of LPS were dialyzed and lyophilized. Dry weights were used to determine the amounts of LPS obtained. The quality of each sample was checked by SDS-polyacrylamide gel electrophoresis (SDS-PAGE).

\section{Formulation of Vaccines}

To use the OMVs or purified LPS as acellular vaccines, vesicle $(3 \mu \mathrm{g})$ and LPS $(1 \mu \mathrm{g})$ preparations were detoxified by mixing with aqueous formaldehyde $(0.37 \%[\mathrm{v} / \mathrm{v}])$ and incubating at $37^{\circ} \mathrm{C}$ overnight, with aluminum hydroxide $(0.2 \mathrm{mg} / \mathrm{mL})$ thereafter being added as an adjuvant.

\section{Active Immunization and Intranasal Challenge}

Four-week-old female BALB/c mice ( $n=12$ per experimental group) obtained from Biol SAIC, Argentina were used for all assays. As described previously (19), the immunization protocols comprised a two-dose schedule (i.p.) with the formulations described above over a period of 2 weeks. Two weeks after the second immunization, the mice were subjected to a nasal challenge with a sublethal dose $\left(10^{6}-10^{7}\right.$ CFUs in $\left.40 \mu \mathrm{L}\right)$ of $B$. parapertussis strain AR729. Then, at 7 days after the challenge, the lungs of the mice were excised and collected for bacterial counts. The number of CFUs was determined as previously described (19). These experiments were carried out in accordance with the recommendations of the Guide for the Care and Use of laboratory animals, National Research Council of National Academies, Washington DC 2010 and/or Directive 2010/63/EU on the protection of animals. The protocol was approved by the Ethical Committee for Animal Experiments of the Faculty of Science at La Plata National University (approval number 003-06-15). 


\section{Enzyme-Linked Immunosorbent Assay (ELISA)}

Plates were coated with sonicated $B$. parapertussis whole-cell lysates in $0.5 \mathrm{M}$ carbonate buffer, $\mathrm{pH} 9.5$ in an overnight incubation at $4{ }^{\circ} \mathrm{C}$ and then blocked with $3 \%(\mathrm{v} / \mathrm{v})$ skimmed milk in blocking buffer $\left(2 \mathrm{~h}\right.$ at $\left.37^{\circ} \mathrm{C}\right)$ before incubation with serially diluted mouse-serum samples $\left(1 \mathrm{~h}\right.$ at $\left.37^{\circ} \mathrm{C}\right)$. The bound IgG was detected after a 2-h incubation with horseradishperoxidase-conjugated goat anti(mouse $\operatorname{IgG}$ ) at a titer of 1:20,000 (Thermo Fisher Scientific, Buenos Aires Argentina). For measuring IgG isotypes, the detection of bound antibody was determined with horseradish-peroxidase-labeled subclassspecific anti(mouse IgG1 or IgG2a) at $1: 8,000$ or 1:1,000, respectively (Sigma Aldrich, USA). The substrate used was 1.0 $\mathrm{mg} / \mathrm{mL}$ o-phenylendiamine (Bio Basic Canada Inc.) in $0.1 \mathrm{M}$ citrate-phosphate buffer, pH 5.0 containing $0.1 \%(\mathrm{v} / \mathrm{v})$ hydrogen peroxide. The optical densities (ODs) were measured with a Titertek Multiskan Model 340 microplate reader (ICN, USA) at $492 \mathrm{~nm}$ and the OD plotted as a function of the log of the reciprocal serum dilution. The inflection point of the curve was determined by the GraphPadPrims ${ }^{\circledR}$ software. Titers were defined as the reciprocal serum dilution giving an $\mathrm{OD}$ corresponding to the inflection point of the curve.

\section{Cell-Line Growth}

Macrophage-like RAW 264.7 cells (ATCC TIB-71) were grown in Roswell Park Memorial Institute (RPMI) 1640 medium containing $10 \%(\mathrm{v} / \mathrm{v})$ heat-inactivated fetal-bovine serum (FBS) and an antibiotic solution of 100 international units (I U)/mL penicillin and $100 \mu \mathrm{g} / \mathrm{mL}$ streptomycin at $37^{\circ} \mathrm{C}$ in $5 \%(\mathrm{v} / \mathrm{v})$ $\mathrm{CO}_{2}$ /air.

\section{Opsonophagocytosis Assay}

A B. parapertussis strain expressing the green-fluorescent protein (GFP)—the B. parapertussis AR729 carrying plasmid pCW504, kindly provided by Dr. A. Weiss-was opsonized by incubation at $37^{\circ} \mathrm{C}$ with different serum samples added at $20 \%(\mathrm{v} / \mathrm{v})$ for $30 \mathrm{~min}$ in a final volume of $40 \mu \mathrm{L}$. Serum-opsonized GFP-expressing bacteria were incubated with RAW 264.7 cells at a multiplicity of infection of 70 for $30 \mathrm{~min}$ at $37^{\circ} \mathrm{C}$ to enable binding and internalization. After extensive washing to remove non-attached bacteria, the samples were analyzed with a FACS Calibur flow cytometer (Becton Dickinson, San Jose, CA) and the results of the estimated degree of phagocytosis expressed as the mean fluorescence intensity. Statistical significance was assessed by the one-way analysis of variance (ANOVA) followed by Bonferroni's multiple-comparison test (GraphPadPrims $\left.{ }^{\circledR}\right)$. Differences were considered to be significant when $p<0.001$.

\section{Bactericidal Assay}

The bactericidal activity of the sera collected from mice 2 weeks after immunization with the OMV(Bpp-LPS-O ${ }^{+}$) and OMV(Bpp-LPS- ${ }^{-}$) vaccines (i.e., the immune sera) and from non-immunized mice (i.e., the naïve sera) were tested in vitro. Naïve sera from mice were used as a source of complement. Both heat-inactivated immune and naïve sera and the vehicle phosphate-buffered saline (PBS) alone were used as controls.
Virulent $B$. parapertussis were grown on Bordet Gengou agar medium and diluted to $1 \times 10^{5} \mathrm{CFUs} / \mathrm{mL}$ in PBS containing $\mathrm{MgCl}_{2} 0.05 \mathrm{M}$ and $\mathrm{CaCl}_{2} 0.15 \mathrm{mM}$. Forty-five microliters of heat inactivated serum or PBS were mixed with $5 \mu \mathrm{L}$ of a suspension containing 500 CFUs of the bacteria. After $1 \mathrm{~h}$ of incubation at $37^{\circ} \mathrm{C}, 5 \mu \mathrm{L}$ of naïve serum was added to each of the samples tested. After $1 \mathrm{~h}$ of incubation at $37^{\circ} \mathrm{C}$, serial dilutions of the samples were spread on Bordet Gengou agar plates and incubated for $48-72 \mathrm{~h}$ to determine the CFUs. At least three biological replicates were performed.

\section{Analysis of the Cellular Response Elicited by Vaccination}

The cellular response was analyzed as previously described $(21,31)$. Briefly, spleen cells from mice immunized with the OMV-based vaccines were harvested 8 weeks after the last immunization and seeded in 48-well culture plates at $10^{6}$ per well in a volume of $500 \mu \mathrm{L}$ of RPMI 1640 cell-culture medium supplemented with $10 \%(\mathrm{v} / \mathrm{v})$ fetal-bovine serum (Invitrogen, Buenos Aires Argentina) containing $100 \mathrm{IU} / \mathrm{mL}$ penicillin and $100 \mu \mathrm{g} / \mathrm{mL}$ streptomycin. All the spleen cells were either stimulated with OMVs derived from B. parapertussis $(5 \mu \mathrm{g} / \mathrm{mL})$ or exposed to medium alone. Supernatants were removed after $72 \mathrm{~h}$ of incubation at $37^{\circ} \mathrm{C}$ in an atmosphere of $5 \%(\mathrm{v} / \mathrm{v}) \quad \mathrm{CO}_{2} /$ air and the production of interferon- $\gamma$ (IFN- $\gamma$ ), and IL-17 determined by ELISA (BD Biosciences, CA USA), according to the conditions specified by the manufacturer.

\section{Adoptive Transfer}

Pooled sera $(100 \mu \mathrm{L})$ or spleen cells $\left(5 \times 10^{7}\right)$ from nonimmunized mice or from mice immunized with the OMVor LPS-based vaccine 2 weeks previously were transferred intraperitoneally to female BALB/c mice $(n=12)$. Twenty-four $\mathrm{h}$ thereafter, the mice were infected with a sublethal dose $\left(10^{6}\right.$ $10^{7}$ CFUs 40 in $\mu \mathrm{L}$ ) of $B$. parapertussis AR729 and the subsequent protection assessed by determining the CFU counts in the mouse lungs 7 days after the challenge.

\section{Statistical Analysis}

The data were evaluated statistically by the Student $t$-test or the ANOVA followed by the Bonferroni post-hoc test (via the GraphPad Prism ${ }^{\circledR}$ software) as indicated in the legends to the figures. Differences were considered significant at a $p$ $<0.05$.

\section{RESULTS}

\section{Isolation and Characterization of the OMVs Obtained From B. parapertussis AR729 and ATCC 15237 Strains}

The OMV samples obtained from B. parapertussis AR729 and B. parapertussis ATCC 15237 grown in the virulent phase were negatively stained and examined by electron microscopy (Figure 1A). The procedure was repeated at least four times, and in all the samples the size range (at dimensions of 50$150 \mathrm{~nm}$ ) was both consistent from batch to batch and similar to 


\section{A}

OMVs (BPp.LPS.O')

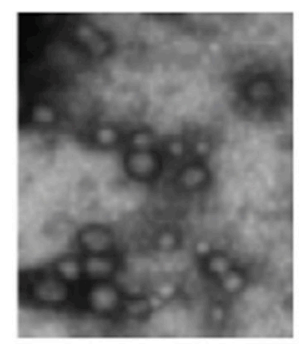

B

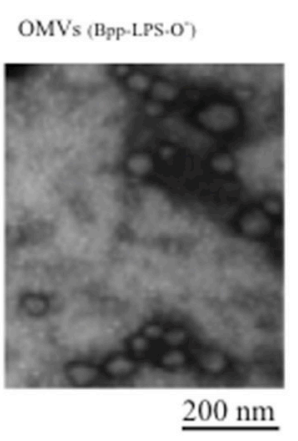

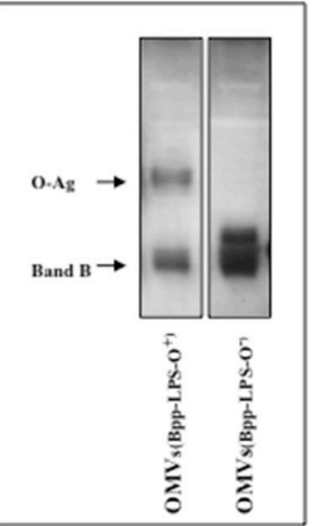

C

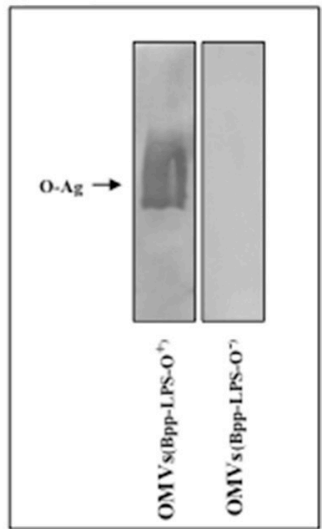

FIGURE 1 | Characterization of OMVs by electron microscopy and electrophoresis. (A) Transmission-electron-microscopy image of the negatively stained preparations obtained in the virulent phase from B. parapertussis strains AR729 (OMVs(Bpp-LPS-O+)) and ATCC 15237 (OMVs(Bpp-LPS-O $\left.{ }^{-}\right)$). Scale bar: 200 nm. (B) LPS analysis of OMVs(Bpp-LPS-O ${ }^{+}$) and OMVs(Bpp-LPS-O- ${ }^{-}$) by $15 \%$ (w/v) SDS-PAGE. The bands were visualized by the BioRad silver-staining technique. In the figure, the sources of the samples are indicated either above (A) or below (B) the representative illustrations. (C) Immunoblotting of purified B. parapertussis LPS separated by $15 \%(\mathrm{w} / \mathrm{v})$ SDS-PAGE probed with the specific $\mathrm{O}$ antigen polyclonal antiserum obtained from mice. The arrows indicate the locations of the $\mathrm{O}$ antigen $(\mathrm{O}-\mathrm{Ag})$ and the Band $\mathrm{B}$.

that previously described for OMV preparations derived from $B$. pertussis (19) and B. parapertussis (13). To characterize the LPS in both OMVs, one-dimensional electrophoresis was performed (Figure 1B). By this technique, we detected that the LPS present in the OMVs preparation derived from B. parapertussis AR729 had the characteristic electrophoretic profile of the LPS belonged from human strains of $B$. parapertussis $(16,32)$ consisting in a faster-migrating LPS $-\mathrm{B}$ band that corresponds to the Lipid A- core structure together with a ladder arrangement of high molecular mass bands that correspond to the $\mathrm{O}$ antigen $(\mathrm{O}-\mathrm{Ag}$, a polymer of 2,3-diacetamido-2,3-dideoxy- $\alpha$-galacturonic acid (33)). The presence of the O-Ag band in the LPS from AR729 was confirmed by an immunoblot assay using a polyclonal serum that only recognized the $\mathrm{O}$ antigen (Figure 1C). In contrast, the LPS from the OMVs derived from the ATCC 15237 strain did not contain the characteristic O-Ag band. Moreover, the polyclonal serum against $\mathrm{O}$ antigen did not react with this LPS derived from ATCC15237 strain (Figure 1C).

\section{Protection Against Intranasal B. parapertussis Challenge After Vaccination With OMVs Obtained From Different Strains}

To evaluate the protective capability against $B$. parapertussis challenge of the OMVs obtained either from $B$. parapertussis AR729 (OMVs(Bpp-LPS-O $\left.{ }^{+}\right)$) or B. parapertussis ATCC 15237 $\left(\mathrm{OMVs}\left(\mathrm{Bpp}-\mathrm{LPS}_{-} \mathrm{O}^{-}\right)\right.$), we used the murine model of intranasal infection ( $n=12$ mice per experimental group). The CFUs recovered from the lungs of the immunized mice were compared with those detected in non-immunized mice (Figure 2). Significant differences were obtained in the lung bacterial counts between the OMVs(Bpp-LPS-O $\left.{ }^{+}\right)$-immunized and non-immunized mice ( $p<0.001$; Figure 2A) and also between the OMVs(Bpp-LPS-O ${ }^{+}$) -immunized and the OMVs(BppLPS- ${ }^{-}$) -immunized mice. The number of colonies recovered from the lungs of the OMVs(Bpp-LPS-O $\left.{ }^{+}\right)$-immunized mice at Day 7 after challenge was at least 4.5 logs lower than those detected in the other two groups of animals included in the assays (Figure 2A).

We next sought to investigate if the different protective capabilities detected for the two OMVs studied here correlated with the corresponding LPSs purified from those OMVs from the two strains of $B$. parapertussis. To that end, we performed in-vivo protection assays using the LPSs isolated from the OMVs derived from each strain, BppLPS- ${ }^{+}$and BppLPS- ${ }^{-}$, as vaccines. Mice ( $n=12$ per each group) were accordingly immunized twice with the purified LPSs $(1 \mu \mathrm{g})$ and then challenged 2 weeks after the second immunization with a sublethal dose of $B$. parapertussis AR729 strain, with non-immunized mice being used as a negative control. For the LPS-O ${ }^{+}$used in the immunization schedule, but not for the LPS-O ${ }^{-}$, highly significant differences of more than 4.5 logs in the lung B. parapertussis colony-formation counts were obtained between the LPS-O ${ }^{+}$immunized animals and the negative control group or the LPS- ${ }^{-}$-immunized animals $(p<0.001$; Figure 2B). Consistent with the role of the $\mathrm{O}$ antigen in conferring immune protection, when the purified LPS-O ${ }^{+}$was added to the formulation based on the OMVs(Bpp-LPS-O ${ }^{-}$), protection against $B$. parapertussis infection was restored to the levels detected for the OMVs(Bpp-LPS-O ${ }^{+}$) vaccine (Figure $2 \mathrm{C}$ ). As would be expected, no such restoration occurred when the same trial was performed with the purified LPS-O ${ }^{-}$(Figure 2C).

Since the B. parapertussis ATCC 15237 failed to colonize the lungsof mice, protection assays using this strain for the challenge were not performed. The failure in colonization of this strain containing an LPS without the $\mathrm{O}$ antigen was expected based on the results reported by Zhang et al. (15). 

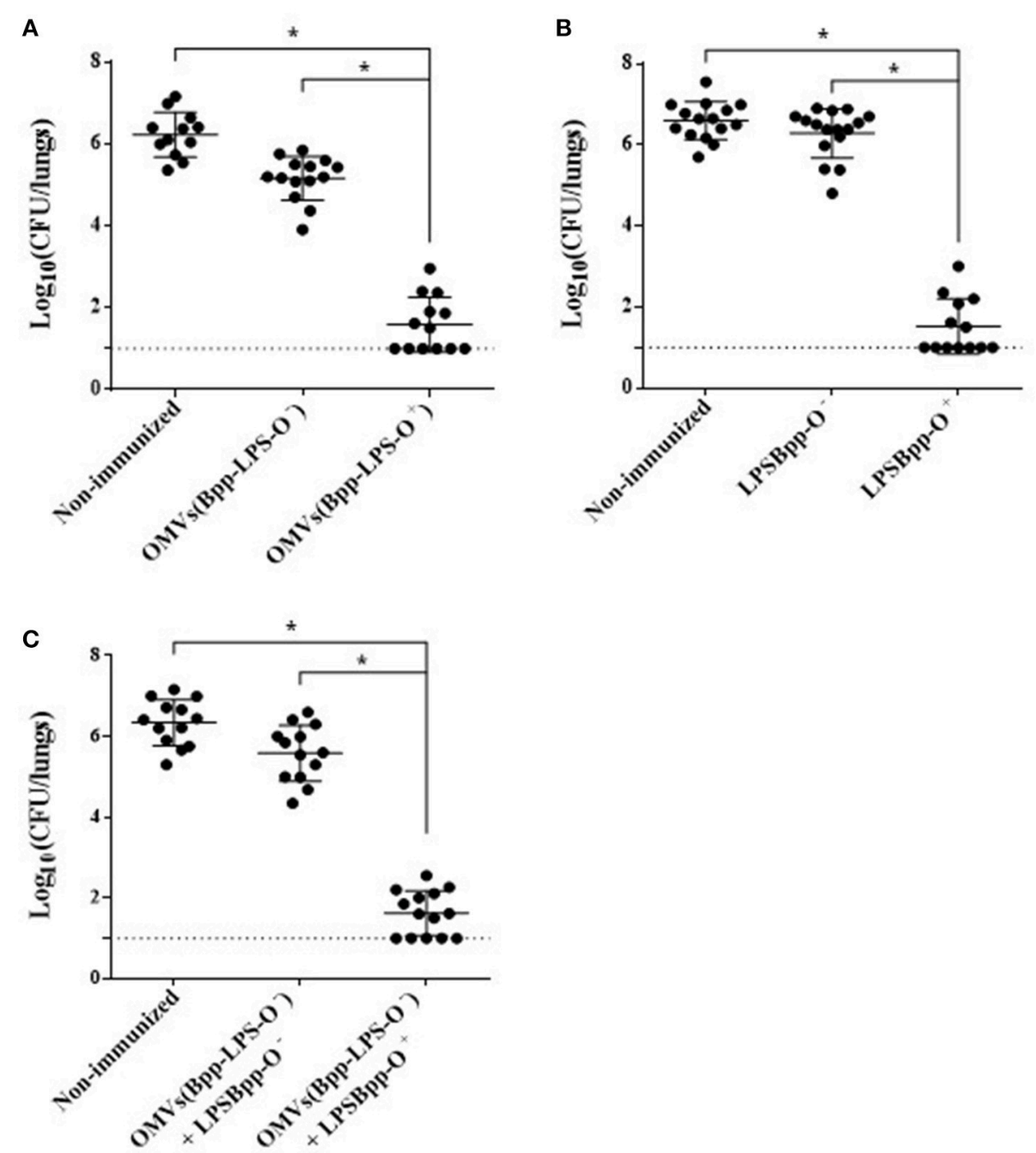

FIGURE 2 | Effect of active immunization on the protection of mice against B. parapertussis infection. (A) Groups of animals were immunized with OMVs(Bpp-LPS-O+ ${ }^{+}$, OMVs(Bpp-LPS-O-) or non-immunized. (B) Groups of animals were immunized with BppLPS-O+ ${ }^{+}$, BppLPS-O- or were non-immunized. (C)

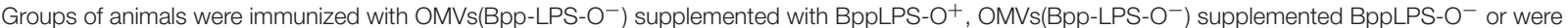
non-immunized. For all panels, the challenge was performed with B. parapertussis AR729 $\left(5 \times 10^{7}\right.$ CFUs in $\left.40 \mu \mathrm{L}\right)$ as the pathogen. In each of the three panels, the number of bacteria recovered from the mouse lungs, expressed as the $\log _{10}$ of the means \pm SEM (error bars) of the CFUs per lungs, is plotted on the ordinate for the samples from the lungs of the experimental groups indicated on the abscissa. Graphs show values for individual mice and the median value (bar, $n=12$ mice) at 7 days after challenge. The dotted horizontal line marks the lower limit of detection. The asterisks (*) indicate significant differences at a $p<0.001$ (ANOVA followed by the Bonferroni post-hoc test).

\section{Key Role of the Humoral Immune Response Induced by the OMVs(Bpp-LPS-O+) That Recognized, Among Others, the $O$ Antigen in the Protection Against B. parapertussis}

To characterize the humoral immune response induced by the OMV based vaccine that induced protection against $B$. parapertussis $\left[\mathrm{OMVs}\left(\mathrm{Bpp}-\mathrm{LPS}-\mathrm{O}^{+}\right)\right]$, we first determined the total IgG and IgG isotypes titers by ELISA assays. The ELISA results showed that mice immunized with OMVs(Bpp-LPS-O $\left.{ }^{+}\right)$ produced high titers of $\operatorname{IgG}(1,460 \pm 210)$ with an IgG1/IgG2a ratio $>1$ (the species $\operatorname{IgG} 1=656 \pm 136$ and $\operatorname{IgG} 2 \mathrm{a}=315 \pm$
83; therefore $\operatorname{IgG} 1 / \operatorname{IgG} 2 \mathrm{a}=2.08)$. Then and based on previously reported role of the $\mathrm{O}-\mathrm{Ag}$ antibodies in protection (15) we wanted to ascertain whether the $\mathrm{LPS}^{-} \mathrm{O}^{+}$embedded in the OMVs(Bpp-LPS-O $\left.{ }^{+}\right)$was able to induce specific antibodies against the $\mathrm{O}-\mathrm{Ag}$ and, as a consequence, the induced immune sera have protective capability against $B$. parapertussis infection. To this end, we performed immunoblotting assays using purified B. parapertussis LPS-O ${ }^{+}$from $B$. parapertussis AR729 strain as the antigen and LPS-O ${ }^{-}$from $B$. parapertussis ATCC 15237 for comparison, and probed against sera from OMVs $\left(\mathrm{Bpp}-\mathrm{LPS}-\mathrm{O}^{+}\right)$ and $\mathrm{OMVs}\left(\mathrm{Bpp}-\mathrm{LPS}^{-} \mathrm{O}^{-}\right.$) immunized mice. Significantly, in this assay the OMVs(Bpp-LPS-O ${ }^{+}$) immune sera recognized a diffuse band that corresponded specifically to the LPS containing the $\mathrm{O}$ 
A

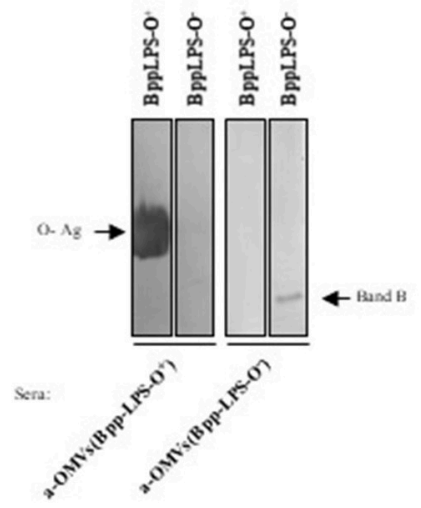

C

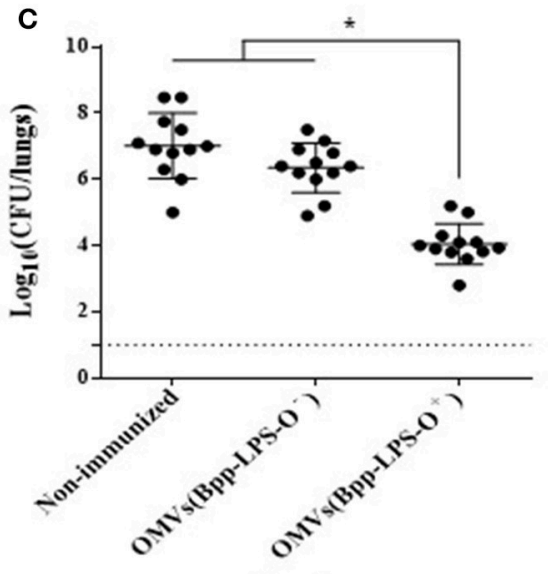

Sera from

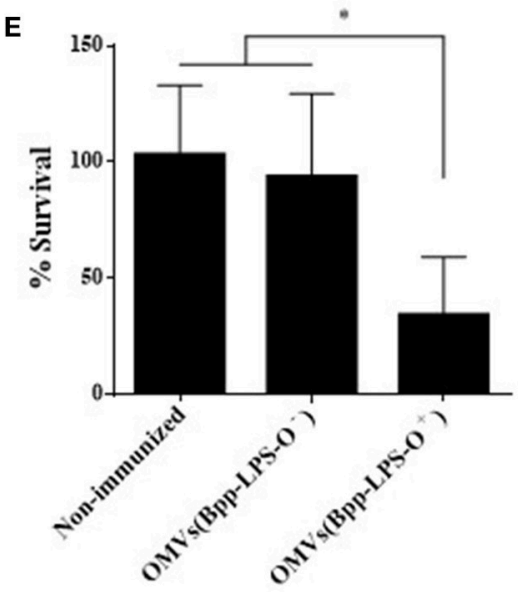

B
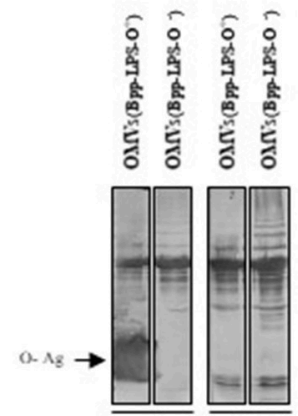

Sera:
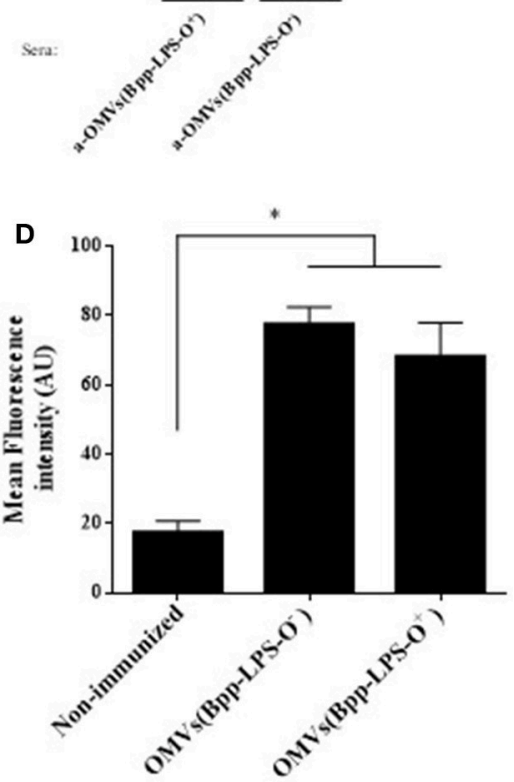

FIGURE 3 | Humoral response induced by purified BppLPS-O+ and OMVs(Bpp-LPS-O+ ${ }^{+}$). (A) Immunoblotting of purified B. parapertussis LPS separated by $15 \%$ $(\mathrm{w} / \mathrm{V})$ SDS-PAGE probed with the polyclonal antiserum obtained from mice immunized with OMVs(Bpp-LPS-O $\mathrm{O}^{+}$) and OMVs(Bpp-LPS-O- $\mathrm{O}^{-}$. The arrows indicate the locations of the $\mathrm{O}$ antigen $(\mathrm{O}-\mathrm{Ag})$ and the Band $\mathrm{B}$ that were recognized by the antiserum used in the subsequent immunoblotting indicated below the gels. These sera are designated according to the immunogen- OMVs(Bpp-LPS-O $\left.{ }^{+}\right)$and $\mathrm{OMVs}\left(\mathrm{Bpp}-\mathrm{LPS}_{-} \mathrm{O}^{-}\right)$-used to raise the immune response in the donor mice. (B) Immunoblotting of OMVs(Bpp-LPS- ${ }^{+}$) and OMVs(Bpp-LPS-O-) samples separated by $12.5 \%(\mathrm{w} / \mathrm{v})$ SDS-PAGE and probed with the polyclonal antiserum obtained from mice immunized with OMVs(Bpp-LPS- $\left.{ }^{+}\right)$and OMVs(Bpp-LPS- $\left.{ }^{-}\right)$. The arrows indicate the locations of the $\mathrm{O}$ antigen $(\mathrm{O}-\mathrm{Ag})$ and the Band $\mathrm{B}$ that were recognized by the antiserum used in the immunoblotting indicated below the gels. These sera are designated according to the immunogen $-\mathrm{OMVs}\left(\mathrm{Bpp}-\mathrm{LPS}-\mathrm{O}^{+}\right.$) and OMVs(Bpp-LPS-O- $-^{-}$- used to raise the immune response in the donor mice. (C) Effect of passive immunization with sera collected from OMVs(Bpp-LPS-O ${ }^{+}$) -

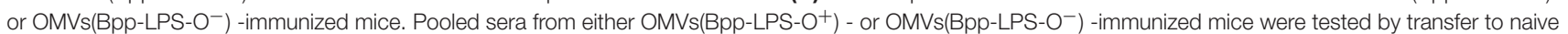


FIGURE 3 | female mice $(n=12)$. Pooled sera from non-immunized mice transferred to naive female mice were used as a negative control of protection. Twenty-four h after the transfer, the mice were infected with B. parapertussis AR729 as the challenge bacterium $\left(5 \times 10^{7} \mathrm{CFUs}\right.$ in $\left.40 \mu \mathrm{L}\right)$. In the figure, the number of bacteria recovered from the mouse lungs, expressed as the $\log _{10}$ of the means \pm SEM (error bars) of the CFUs per lungs, is plotted on the ordinate for the non-immune and the two immune sera indicated on the abscissa, with the latter being designated according to the immunogen-OMVs(Bpp-LPS-O ${ }^{-}$) and OMVs(Bpp-LPS-O ${ }^{+}$) - used to raise the immune response in the donor mice. The asterisk $\left(^{\star}\right)$ indicates a significant difference vs. the naïve sera ( $\left.p \leq 0.05\right)$. (D) Opsonophagocytic activiy of the immune sera. The mean fluorescence intensity in arbitrary units (AU) associated with macrophages previously incubated with GFP-expressing B. parapertussis AR729 is plotted on the ordinate for each of the sera used for opsonization of those bacteria on the abscissa, indicated as non-immune or immune, with the latter being designated according to the immunogen-OMVs(Bpp-LPS-O ${ }^{-}$) and OMVs(Bpp-LPS-O $\left.{ }^{+}\right)$- used to raise the immune response in the donor mice. The asterisk $\left.{ }^{*}\right)$ denotes $p \leq 0.0001$ vs. the intensity obtained after exposure of the bacteria to the non-immunized serm. (E) Serum-complement-mediated killing. Sera from $\mathrm{OMVs}\left(\mathrm{Bpp}-\mathrm{LPS}_{-} \mathrm{O}^{+}\right.$) - or OMVs(Bpp-LPS-O ${ }^{-}$)-immunized mice were incubated with B. parapertussis AR729 (500 CFU), and $1 \mathrm{~h}$ later the bacteria were plated to determine viability. In the figure, the percent survival is plotted on the ordinate for the non-immune and the two immune sera indicated on the abscissa, with the latter being designated according to the immunogen-OMVs(Bpp-LPS-O ${ }^{-}$) and OMVs(Bpp-LPS-O $\left.{ }^{+}\right)$- used to raise the immune response in the donor mice. The asterisk $\left(^{\star}\right)$ indicates a significant difference vs. the naïve sera ( $\left.p \leq 0.05\right)$.

antigen (Figure 3A, left lane). This band was not detected in the O-antigen-deficient LPS isolated from the B. parapertussis ATCC 15237 strain (Figure 3A). In contrast, the sera from OMVs(BppLPS- $\mathrm{O}^{-}$)-treated mice recognized only the fast-migrating Band B (Figure 3A, right lane). Naïve sera did not react with either of the bands detected in the SDS-PAGE for both LPSs (not shown). When the OMV-generated immune sera were used in immunoblotting assays, in which the OMVs from both the $\mathrm{O}$-antigen-positive and -negative $B$. parapertussis strains were used as antigens, the polypeptide profile recognized was similar for both sera. The major difference observed between the two sera and the two OMV preparations, was the diffuse band corresponding to the $\mathrm{O}$ antigen detected in the OMVs(BppLPS-O ${ }^{+}$) sample by the OMVs $\left(B p p-L P S-O^{+}\right.$) sera (Figure 3B, leftmost lane).

With the immune sera induced by OMVs(Bpp-LPS-O $\left.{ }^{+}\right)$ having been characterized in this manner, serum-transfer experiments were performed. The sera to be tested were adoptively injected into naïve animals $(n=12) 24 \mathrm{~h}$ before an intranasal challenge with $B$. parapertussis $\left(5 \times 10^{7}\right.$ CFUs B. parapertussis AR729). Sera from OMVs(Bpp-LPS-O ${ }^{-}$) immunized mice and from naïve mice were used as negative control of protection. Through this assay, we detected that B. parapertussis was cleared from the mouse lungs by Day 7 after the inoculation with the OMVs(Bpp-LPS-O $\left.{ }^{+}\right)$sera containing anti-O Ag antibodies (at a reduction of 4 logs), whereas the OMVs(Bpp-LPS-O ${ }^{-}$) or naïve sera in which anti$\mathrm{O}$ Ag antibodies were not detected had no significant effect (Figure 3C).

To elucidate the mechanisms involved in the protective capacity of the OMVs(Bpp-LPS-O ${ }^{+}$) immune sera, we decided to perform an oposonophagocytosis assay since this activity was previously associated with the presence of antibodies against the $B$. parapertussis $\mathrm{O}$ antigen (15). Consistent with the ELISA results demonstrating that mice immunized with OMVs(Bpp-LPS-O ${ }^{+}$) produced high titers of IgG with an IgG1/IgG2a ratio $>1, B$. parapertussis was efficiently opsonized by the antibodies from OMVs(Bpp-LPS-O ${ }^{+}$) -induced serum (Figure 3D). We need to remark however, that the immune sera from OMVs(Bpp-LPS-O ${ }^{-}$) -treated mice also presented oposonophagocytic activity. These results are not surprising since other opsonins are expected to be induced by OMVs because of the wide variety of immunogens present. No significant internalization was detected for bacteria opsonized with naïve serum (Figure 3D).

To determine whether the different degree of protection observed for the two OMV vaccines was correlated with a differential bactericidal capability between the sera induced by both OMVs, killing assays were performed. For these determinations, a suspension of 500 CFUs of B. parapertussis bacteria was incubated in $50 \mu \mathrm{L}$ of $90 \%(\mathrm{v} / \mathrm{v})$ serum in PBS to insure that the serum components were not limiting. These assays revealed that the $B$. parapertussis bacteria were sensitive to the immune sera induced by the OMVs(Bpp-LPS-O $\left.{ }^{+}\right)$, with only $24.6 \pm 10.2 \%$ surviving, but resistant to OMVs(Bpp-LPS-O ${ }^{-}$) and naïve sera, with respective percent survivals of $92.8 \pm 20.5$ and $92.8 \pm 11.2$ (Figure 3E). In control experiments using heatinactivated sera, we observed a $100 \%$ bacterial survival for both the immune and the naïve sera.

All the results presented here indicated that the presence of $\mathrm{O}-\mathrm{Ag}$ embedded in the OMVs(Bpp-LPS-O ${ }^{+}$) induced humoral immune responses that conferred a definitive protection against B. parapertussis. The protective capability observed seems to be mainly a consequence of the bactericidal activity of the immune sera.

To investigate whether the immune spleen cells, and more specifically the T-cells, were also involved in the immunogenicity and protection conferred by the OMVs(Bpp$\mathrm{LPS}_{-} \mathrm{O}^{+}$) formulation, we performed spleen-cell-activation and -proliferation assays and adoptive-transfer experiments. The results of the activation assays (Figure 4) revealed that 2 months after immunization, OMVs(Bpp-LPS-O ${ }^{+}$) vaccination in contrast to non-immunized mice induced high concentrations of IFN- $\gamma(844 \pm 59 \mathrm{pg} / \mathrm{mL}$; Figure 4A) and IL-17 (244 $\pm 61 \mathrm{pg} / \mathrm{mL}$; Figure 4A). Similar data for both cytokines were detected in mice immunized with OMVs(Bpp-LPS$\mathrm{O}^{-}$) (Figure 4A). These findings strongly indicated that vaccination with any of the two OMV vaccines used here, induced a mixed Th1-Th17 spleen-cell immune-response profile.

To perform adoptive-transfer assays, naïve BALB/c mice were treated either with $5 \times 10^{7}$ intact spleen cells from mice that had been immunized with OMVs(Bpp-LPS-O $\left.{ }^{+}\right)$or OMVs(Bpp$\mathrm{LPS}^{-} \mathrm{O}^{-}$) vaccine 2 weeks before or with spleen cells from nonimmunized animals. Twenty-four h later, the mice $(n=12$ per group) were infected with $5 \times 10^{7}$ CFUs of $B$. parapertussis AR729 


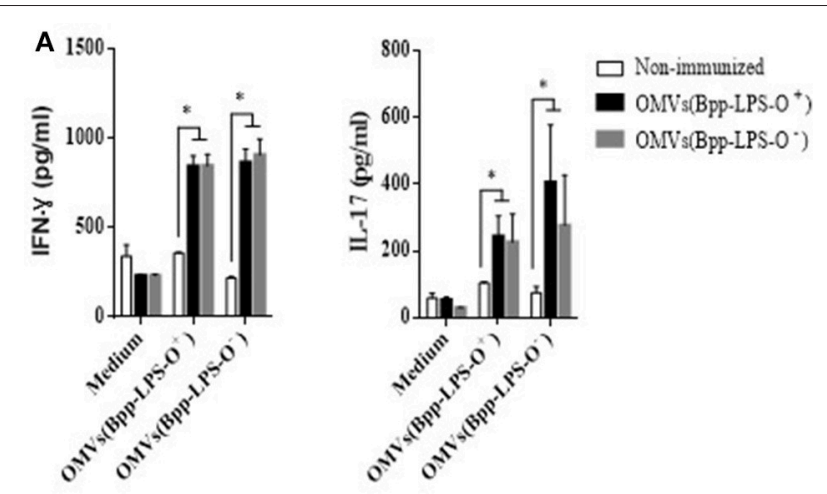

B

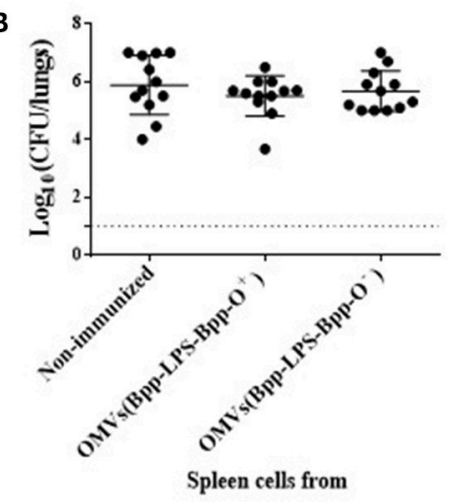

FIGURE 4 | Immune response induced by active immunization with OMVs(Bpp-LPS-O ${ }^{+}$) vaccine. (A) Cytokine production by spleen cells from immunized mice. BALB/c mice were non-immunized or immunized with two doses of OMVs(Bpp-LPS-O ${ }^{+}$) or OMVs(Bpp-LPS-O $\left.{ }^{-}\right)$vaccine. Two months after the last immunization, mice were sacrificed and their spleen cells exposed to OMVs(Bpp-LPS-O+ $)$, OMVs(Bpp-LPS-O- $)$ or to medium alone (negative control). After $72 \mathrm{~h}$ of culture, the concentrations of IFN- $\gamma$ and IL-17 (A) were determined in the culture supernatant by ELISA. In both cases, the concentration of cytokine in $\mathrm{pg} / \mathrm{mL}$ is plotted on the ordinate for exposure of spleen cells from non-immunized mice (white bars) or mice immunized with OMVs(Bpp-LPS-O ${ }^{+}$) (black bars) or with OMVs(Bpp-LPS-O ${ }^{-}$) (gray bars) to either medium alone, medium + OMVs(Bpp-LPS-O $\left.{ }^{+}\right)$or medium + OMVs(Bpp-LPS-O- ${ }^{-}$, as indicated on the abscissa. The results are expressed as mean values ( \pm standard error) ( $n=12$ mice per group). Significant differences were analyzed for each cytokine between non-immunized and immunized mice ( ${ }^{*} p \leq 0.01$, ANOVA followed by the Bonferroni post-hoc test). (B) Effect of passive immunization with spleen cells collected from OMVs(Bpp-LPS-O ${ }^{+}$) or OMVs(Bpp-LPS-O-) -immunized mice. Whole spleen cells $\left(2-5 \times 10^{7}\right)$ from OMV-immunized or non-immunized mice were tested as possible vehicles of immune protection by passive transfer to naïve mice ( $n$ $=12$ per group). Twenty-four $h$ after transfer, the mice were infected with $B$. parapertussis AR729 $\left(5 \times 10^{7} \mathrm{CFU} / 40 \mu \mathrm{L}\right)$. In the panel, the $\log _{10}$ means \pm SEM (error bars) of bacteria per lungs is plotted on the ordinate for mice receiving spleen cells from non-immunized donor mice or from those immunized with OMVs(Bpp-LPS-O+ ${ }^{+}$) or with OMVs(Bpp-LPS-O- ${ }^{-}$), as indicated on the abscissa. The data are the means of 12 mice per group at 7 days postchallenge. The dotted horizontal line marks the lower limit of detection. The results were analyzed by ANOVA followed by the Bonferroni post-hoc test.

and then sacrificed 7 days later to determine the number of CFU counts in the lungs (Figure 4B). The transfer of spleen cells from immunized animals with any of the two OMV vaccines used here resulted in a non-reduction of bacterial colonization, with the CFU counts in the animals that received immune or naïve spleen cells being statistically indistinguishable.

These results indicate that spleen cell-mediated immunity would appear not to play a significant role in the protection against $B$. parapertussis induced by the OMVs(Bpp-LPS-O ${ }^{+}$) vaccine.

\section{Protection Against Intranasal B. pertussis Challenge After Vaccination With OMV From B. pertussis Supplemented With Purified B. parapertussis LPS-O}

In view of the protective capabilities of OMVs derived from B. pertussis (OMVsBp) against B. pertussis infection and of the purified $B$. parapertussis $\mathrm{LPS}^{-} \mathrm{O}^{+}$against $B$. parapertussis challenge, we wanted to evaluate whether a combined experimental vaccine containing OMVsBp plus purified LPS-O ${ }^{+}$was able to confer a simultaneous protection against both pathogen infections at a comparable level with respect to that of the individual vaccines (OMVsBp vaccine and $\mathrm{LPS}_{-} \mathrm{O}^{+}$vaccine). To that end, we analyzed the effect of a double-dose-immunization schedule using a preparation containing OMVsBp plus the purified B. parapertussis LPS$\mathrm{O}^{+}$on the subsequent lung colonization of mice challenged with B. pertussis Tohama phase I strain or B. parapertussis AR729 $\left(\approx 10^{7}\right.$ CFUs in $40 \mu \mathrm{L}$; Figure 5A,B $)$. The results were compared with those obtained for mice that had been immunized with each of the two vaccines- OMVsBp and BppLPS-O ${ }_{-}^{+}$ exclusively (Figure 5). PBS-injected mice served as a negative control. Significant differences in the lung bacterial counts between the immunized animals and the negative control group were observed ( $p<0.05$; Figure 5). The protection induced by a OMVsBp plus purified LPS-O ${ }^{+}$vaccine against $B$. pertussis challenge reached the same level as that induced by the OMVsBp immunization alone, with the number of CFUs recovered from the lungs at Day 7 after the challenge likewise dropping by at least 3 orders of magnitude compared to that of the non-immunized mice (Figure 5A). Similar results were observed conversely for $B$. parapertussis challenge: the number of $B$. parapertussis CFUs recovered from the lungs at Day 7 after challenge likewise dropped by at least 3 orders of magnitude in mice immunized either with OMVsBp plus purified LPS-O ${ }^{+}$vaccine or with LPS$\mathrm{O}^{+}$vaccine alone compared to the level of infection occurring in the non-immunized mice (Figure 5B).

The results presented in this section thus demonstrated that the vaccines based on OMVs of $B$. pertussis and on the LPS-O ${ }^{+}$ of $B$. parapertussis could be combined without altering the levels of protection inducible by each one independently.

\section{DISCUSSION}

We recently identified a novel vaccine candidate against $B$. parapertussis consisting of the bacterial outer-membrane vesicles (13). In previous work we had presented results on the protection conferred by this OMV-based vaccine formulation against $B$. 

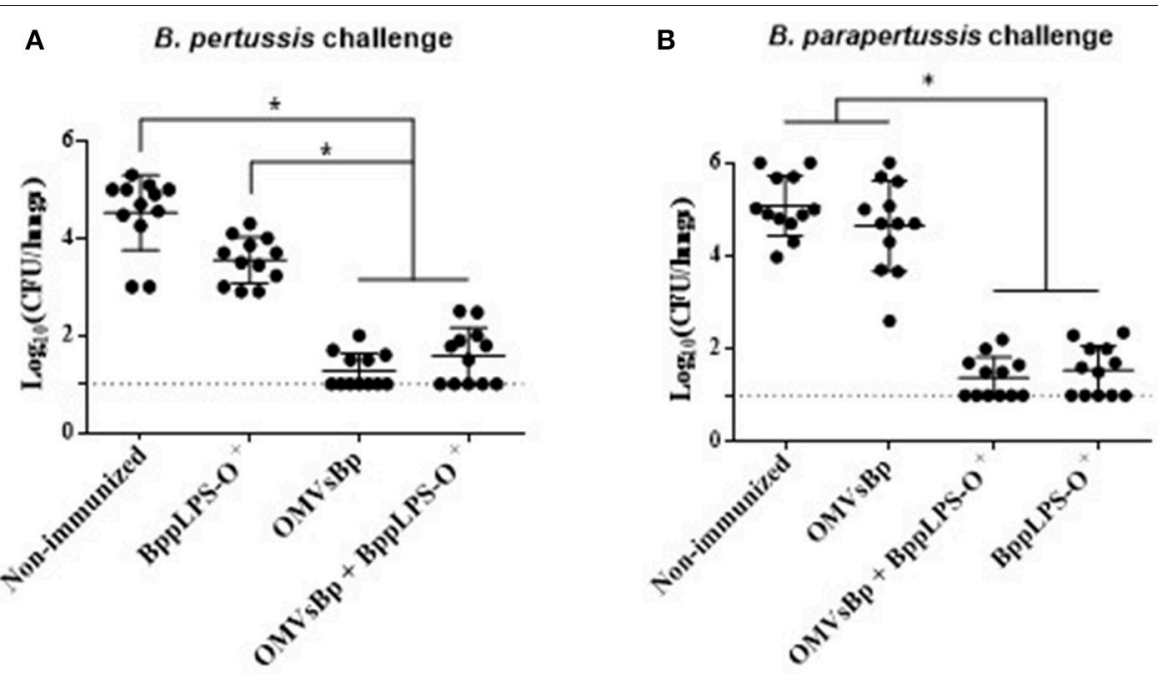

FIGURE 5 | Effect of active intraperitoneal immunization with OMVsBp and/or BppLPS-O + vaccine in the mouse-intranasal-challenge model. Female mice $(n=12$ per treatment) were vaccinated twice with a OMVsBp + BppLPS-O ${ }^{+}$vaccine at a 2-week interval. Immunization with OMVs derived from B. pertussis or the LPS-O ${ }^{+}$ from B. parapertussis alone were used as controls. (A) B. pertussis Tohama was used as the challenge bacterium (5 $\times 10^{7} \mathrm{CFUs}$ in $\left.40 \mu \mathrm{L}\right)$. (B) $B$. parapertussis AR729 was used as challenge bacterium $\left(5 \times 10^{7}\right.$ CFUs in $\left.40 \mu \mathrm{L}\right)$. In both panels, the number of bacteria recovered from the mouse lungs, expressed as the $\log _{10}$ (CFUs) per lungs \pm SEM (error bars) is plotted on the ordinates for non-immunized mice or for those immunized with a vaccine containing OMVsBp and/or the O-antigen-containing lipopolysaccharide LPS-O+, as indicated on the abscissa. Graphs show values for individual mice and the median value (bar, $n=12$ mice) at 7 days after challenge. The dotted horizontal line marks the lower limit of detection. The asterisks $\left(^{*}\right)$ indicate significant differences at a $p<0.05$ (ANOVA followed by the Bonferroni post-hoc test).

parapertussis but also against infections caused by $B$. pertussis. In view of the results of Zhang and colleagues on the protective capability of $B$. parapertussis LPS containing the $\mathrm{O}$ antigen in its structure (15), we decided to analyze whether OMVs containing this LPS embedded within possessed the same ability to confer protection and, if so, to analyze to what extent that component was responsible for the protective capability $(15,34)$. In order to achieve these objectives, we carried out comparative studies with a strain of $B$. parapertussis in which we detected the presence of an LPS lacking the $\mathrm{O}$ antigen. This detection was potentially both relevant and informative because that strain ATCC 15237 of $B$. parapertussis was isolated by Kendrick and Eldering in 1938 from a human patient with symptoms similar to pertussis (25). To the best of our knowledge, that finding constituted the first description of a human isolate in which the $\mathrm{O}$ antigen was not detected. This finding allowed us to perform comparative protection assays, using the intranasal-challenge model in mice, with vaccines based on vesicles derived from the Argentine strain containing the LPS with the $\mathrm{O}$ antigen and the ATCC strain lacking that $\mathrm{O}$ antigen. These assays enabled us to ascertain the role of the vesicle LPS with $\mathrm{O}$ antigen in conferring protection. Thus, while the OMVsBpp containing the LPS with the $\mathrm{O}$ antigen adequately protected mice against $B$. parapertussis infection, the presence of LPS without that key antigen in the OMVs was unable to attain the levels of protection afforded by the OMVs(Bpp-LPS$\mathrm{O}^{+}$) -based immunogen. The differential protective capability associated with the presence of the $\mathrm{O}$ antigen was confirmed with preparations of purified $\mathrm{LPS}_{-} \mathrm{O}^{+}$or $\mathrm{LPS}^{-} \mathrm{O}^{-}$as vaccines. Furthermore, the $\mathrm{LPS}^{-\mathrm{O}^{+}}$provided a protection against $B$. parapertussis infections even when combined with OMVsBpp containing LPS devoid of the $\mathrm{O}$ antigen or with OMVs derived from $B$. pertussis which was previously described as an atractive vaccine candidate againt $B$. pertussis infection containing an important number of protective immunogens (20, 22, 23). Of particular significance in that last formulation combining the OMVs of $B$. pertussis with the LPS-O ${ }^{+}$from B. parapertussis was the ability to protect mice against infections caused by either Bordetella species-a feature of considerable relevance to pertussis epidemiology with respect to both $B$. pertussis and $B$. parapertussis.

The results presented here demonstrate that $\mathrm{LPS}^{-} \mathrm{O}^{+}$is a protective immunogen even within the internal environment of OMVsBpp.

Antibodies raised against $B$. parapertussis LPS-O $^{+}$or OMVs(Bpp-LPS-O ${ }^{+}$) efficiently cleared B. parapertussis from mouse lungs (Figure 3). In contrast, the immune sera from $B$. parapertussis LPS lacking the $\mathrm{O}$ antigen and from OMVs(BppLPS- ${ }^{-}$) are much less effective in killing the bacteria in vitro or in mediating bacterial clearance in vivo than sera induced by the formulations containing $B$. parapertussis vesicles with LPS-O+ (Figure 3).

Regarding the bactericidal activity of naïve sera and the sensitivity of $B$. parapertussis to the non-immune complement, the ovine $B$. parapertussis lacking the $\mathrm{O}$ antigen was previously reported to be highly sensitive to murine complement-mediated killing in vitro (18). Conversely, the $\mathrm{O}$ antigen was found to enable $B$. parapertussis to efficiently colonize the lower respiratory tract by protecting against complement-mediated control and clearance (35). The results presented here are in agreement with previous reports $(18,35)$ demonstrating that $B$. 
parapertussis strains harboring the $\mathrm{O}$ antigen were resistant not only to naïve sera but also to the sera induced by the OMVs(BppLPS- ${ }^{-}$) (Figure 3F). In contrast, Wolfe et al demonstrated that the complement of immune animals is required for the killing function of serum antibodies against B. parapertussis (36). The present study of OMVs(Bpp-LPS-O ${ }^{+}$) -antibody-mediated clearance of $B$. parapertussis furthermore suggests that antibodyopsonized bacteria, in fact, activate the complement cascade, a mechanism of clearance that is in agreement with the one proposed by Wolfe et al. (36).

In these experiments, we also detected that the OMVs derived from $B$. parapertussis, as likewise occurs with those derived from $B$. pertussis $(21,31)$, were able to induce a Th1-Th17 profile. Nevertheless, in contrast to the OMVs derived from $B$. pertussis (31), we observed that immune spleen cells do not contribute to the protection exhibited by the OMVBpp-based vaccine: rather, that vaccine seems to protect against $B$. parapertussis infection mainly through the induction of a humoral immune response, as was evidenced by the adoptive-transfer experiments. In conclusion, the body of this work has demonstrated that the $\mathrm{O}$ antigen constitutes a crucial immunogen-indeed, a sine qua non-in the formulation of the OMVBpp-based vaccine.

\section{REFERENCES}

1. Lautrop H. Epidemics of parapertussis. 20 years' observations in Denmark. Lancet (1971) 1:1195-8.

2. Heininger U, Stehr K, Christenson P, Cherry JD. Evidence of efficacy of the Lederle/Takeda acellular pertussis component diphtheria and tetanus toxoids and pertussis vaccine but not the Lederle whole-cell component diphtheria and tetanus toxoids and pertussis vaccine against Bordetella parapertussis infection. Clin Infect Dis. (1999) 28:602-4. doi: 10.1086/515154

3. Cherry JD. Why do pertussis vaccines fail? Pediatrics (2012) 129:968-70. doi: 10.1542/peds.2011-2594

4. Watanabe M, Nagai M. Whooping cough due to Bordetella parapertussis: an unresolved problem. Expert Rev Anti Infect Ther. (2004) 2:447-54. doi: 10.1586/14787210.2.3.447

5. Koepke R, Bartholomew ML, Eickhoff JC, Ayele RA, Rodd D, Kuennen J, et al. Widespread Bordetella parapertussis infections-wisconsin, 2011-2012: clinical and epidemiologic features and antibiotic use for treatment and prevention. Clin Infect Dis. (2015) 61:1421-31. doi: 10.1093/cid/civ514

6. Cherry JD, Seaton BL. Patterns of Bordetella parapertussis respiratory illnesses: 2008-2010. Clin Infect Dis. (2012) 54:534-7. doi: 10.1093/cid/cir860

7. David S, van Furth R, Mooi FR. Efficacies of whole cell and acellular pertussis vaccines against Bordetella parapertussis in a mouse model. Vaccine (2004) 22:1892-8. doi: 10.1016/j.vaccine.2003.11.005

8. Khelef N, Danve B, Quentin-Millet MJ, Guiso N. Bordetella pertussis and Bordetella parapertussis: two immunologically distinct species. Infect Immun. (1993) 61:486-90.

9. Liko J, Robison SG, Cieslak PR. Do pertussis vaccines protect against Bordetella parapertussis? Clin Infect Dis. (2017) 64:1795-7. doi: $10.1093 / \mathrm{cid} / \mathrm{cix} 221$

10. Long GH, Karanikas AT, Harvill ET, Read AF, Hudson PJ. Acellular pertussis vaccination facilitates Bordetella parapertussis infection in a rodent model of bordetellosis. Proc Biol Sci. (2010) 277:2017-25. doi: 10.1098/rspb.2010.0010

11. Komatsu E, Yamaguchi F, Eguchi M, Watanabe M. Protective effects of vaccines against Bordetella parapertussis in a mouse intranasal challenge model. Vaccine (2010) 28:4362-8. doi: 10.1016/j.vaccine.2010.04.053

12. Alvarez Hayes J, Oviedo JM, Valdez H, Laborde JM, Maschi F, Ayala $\mathrm{M}$, et al. A recombinant iron transport protein from Bordetella pertussis

\section{AUTHOR CONTRIBUTIONS}

DH planned the study, made the laboratory analysis, interpreted data, and drafted manuscript. DB, MG, and MZ planned the study, interpreted data, and revised figures and the manuscript. $\mathrm{ME}, \mathrm{EB}, \mathrm{FC}, \mathrm{PM}$, and $\mathrm{CC}$ performed certain experiments and laboratory analyses. All authors approved the final manuscript.

\section{FUNDING}

This work was supported by ANCPyT (PICT 2014-3617, PICT 2012- 2719), CONICET and FCE-UNLP (Argentina) grants to DH. DH, MG, MZ, and DB, are members of the Scientific Career of CONICET. FC and EB are fellows from CONICET. PM and $\mathrm{ME}$ are fellows from ANPCyT and CC is a full-time Professor at Universidad Nacional de La Plata.

\section{ACKNOWLEDGMENTS}

Luciana Cayuela provided excellent technical assistance. Dr. Donald F. Haggerty, a retired academic career investigator and native English speaker, edited the final version of the manuscript.

confers protection against Bordetella parapertussis. Microbiol Immunol. (2017) 61:407-15. doi: 10.1111/1348-0421.12532

13. Bottero D, Gaillard ME, Errea A, Moreno G, Zurita E, Pianciola L, et al. Outer membrane vesicles derived from Bordetella parapertussis as an acellular vaccine against Bordetella parapertussis and Bordetella pertussis infection. Vaccine (2013) 31:5262-8. doi: 10.1016/j.vaccine.2013.08.059

14. Kulp A, Kuehn MJ. Biological functions and biogenesis of secreted bacterial outer membrane vesicles. Annu Rev Microbiol. (2010) 64:163-84. doi: 10.1146/annurev.micro.091208.073413

15. Zhang X, Goebel EM, Rodriguez ME, Preston A, Harvill ET. The O antigen is a critical antigen for the development of a protective immune response to Bordetella parapertussis. Infect Immun. (2009) 77:5050-8. doi: 10.1128/IAI.00667-09

16. Caroff M, Aussel L, Zarrouk H, Martin A, Richards JC, Therisod H, et al. Structural variability and originality of the Bordetella endotoxins. J. Endotoxin Res. (2001) 7:63-8. doi: 10.1177/09680519010070011101

17. Marr N, Tirsoaga A, Blanot D, Fernandez R, Caroff M. Glucosamine found as a substituent of both phosphate groups in Bordetella lipid A backbones: role of a BvgAS-activated ArnT ortholog. J. Bacteriol. (2008) 190:4281-90. doi: 10.1128/JB.01875-07

18. Hester SE, Goodfield LL, Park J, Feaga HA, Ivanov YV, Bendor L, et al. Host specificity of ovine Bordetella parapertussis and the role of complement. PLoS ONE (2015) 10:e0130964. doi: 10.1371/journal.pone.0130964

19. Asensio CJ, Gaillard ME, Moreno G, Bottero D, Zurita E, Rumbo M, et al. Outer membrane vesicles obtained from Bordetella pertussis Tohama expressing the lipid A deacylase PagL as a novel acellular vaccine candidate. Vaccine (2011) 29:1649-56. doi: 10.1016/j.vaccine.2010.12.068

20. Roberts R, Moreno G, Bottero D, Gaillard ME, Fingermann M, Graieb A, et al. Outer membrane vesicles as acellular vaccine against pertussis. Vaccine (2008) 26:4639-46. doi: 10.1016/j.vaccine.2008.07.004

21. Gaillard ME, Bottero D, Errea A, Ormazabal M, Zurita ME, Moreno G, et al. Acellular pertussis vaccine based on outer membrane vesicles capable of conferring both long-lasting immunity and protection against different strain genotypes. Vaccine (2014) 32:931-7. doi: 10.1016/j.vaccine.2013.12.048

22. Hozbor DF. Outer membrane vesicles: an attractive candidate for pertussis vaccines. Expert Rev Vaccines (2016) 16:193-6. doi: $10.1080 / 14760584.2017 .1276832$ 
23. Gasperini G, Biagini M, Arato V, Gianfaldoni C, Vadi A, Norais N, et al. Outer Membrane Vesicles (OMV)-based and proteomics-driven antigen selection identifies novel factors contributing to Bordetella pertussis adhesion to epithelial cells. Mol Cell Proteomics (2018) 17:205-15. doi: 10.1074/mcp.RA117.000045

24. Raeven RH, Brummelman J, Pennings JL, van der Maas L, Tilstra W, Helm K, et al. Bordetella pertussis outer membrane vesicle vaccine confers equal efficacy in mice with milder inflammatory responses compared to a whole-cell vaccine. Sci Rep. (2016) 6:38240. doi: 10.1038/srep38240

25. Eldering G, Kendrick P. Bacillus Para-Pertussis: a species resembling both bacillus pertussis and bacillus bronchisepticus but identical with neither. $J$ Bacteriol. (1938) 35:561-72.

26. Stainer DW, Scholte MJ. A simple chemically defined medium for the production of phase I Bordetella pertussis. J Gen Microbiol. (1970) 63:211-20. doi: 10.1099/00221287-63-2-211

27. Hozbor D, Rodriguez ME, Fernandez J, Lagares A, Guiso N, Yantorno O. Release of outer membrane vesicles from Bordetella pertussis. Curr Microbiol. (1999) 38:273-8. doi: 10.1007/PL00006801

28. Bradford MM. A rapid and sensitive method for the quantitation of microgram quantities of protein utilizing the principle of protein-dye binding. Anal Biochem. (1976) 72:248-54. doi: 10.1016/0003-2697(76) 90527-3

29. Laemmli UK. Cleavage of structural proteins during the assembly of the head of bacteriophage T4. Nature (1970) 227:680-5. doi: 10.1038/227680a0

30. Hozbor D, Rodriguez ME, Samo A, Lagares A, Yantorno O. Release of lipopolysaccharide during Bordetella pertussis growth. Res Microbiol. (1993) 144:201-9. doi: 10.1016/0923-2508(93)90045-4

31. Bottero D, Gaillard ME, Zurita E, Moreno G, Martinez DS, Bartel E, et al. Characterization of the immune response induced by pertussis OMVs-based vaccine. Vaccine (2016) 34:3303-9. doi: 10.1016/j.vaccine.2016.04.079
32. Hittle LE, Jones JW, Hajjar AM, Ernst RK, Preston A. Bordetella parapertussis PagP mediates the addition of two palmitates to the lipopolysaccharide lipid A. J Bacteriol. (2015) 197:572-80. doi: 10.1128/JB.02236-14

33. Di Fabio JL, Caroff M, Karibian D, Richards JC, Perry MB. Characterization of the common antigenic lipopolysaccharide O-chains produced by Bordetella bronchiseptica and Bordetella parapertussis. FEMS Microbiol Lett. (1992) 76:275-81. doi: 10.1111/j.1574-6968.1992.tb05475.x

34. Zhang X, Rodriguez ME, Harvill ET. O antigen allows B. parapertussis to evade B. pertussis vaccine-induced immunity by blocking binding and functions of cross-reactive antibodies. PLoS ONE (2009) 4:e6989. doi: 10.1371/journal.pone.0006989

35. Goebel EM, Wolfe DN, Elder K, Stibitz S, Harvill ET. O antigen protects Bordetella parapertussis from complement. Infect Immun. (2008) 76:1774-80. doi: 10.1128/IAI.01629-07

36. Wolfe DN, Kirimanjeswara GS, Harvill ET. Clearance of Bordetella parapertussis from the lower respiratory tract requires humoral and cellular immunity. Infect Immun. (2005) 73:6508-13. doi: 10.1128/IAI.73.10.6508-6513.2005

Conflict of Interest Statement: The authors declare that the research was conducted in the absence of any commercial or financial relationships that could be construed as a potential conflict of interest.

Copyright (c) 2018 Bottero, Zurita, Gaillard, Carriquiriborde, Martin Aispuro, Elizagaray, Bartel, Castuma and Hozbor. This is an open-access article distributed under the terms of the Creative Commons Attribution License (CC BY). The use, distribution or reproduction in other forums is permitted, provided the original author(s) and the copyright owner(s) are credited and that the original publication in this journal is cited, in accordance with accepted academic practice. No use, distribution or reproduction is permitted which does not comply with these terms. 